\title{
BITTERER GESCHMACK ALS WARNZEICHEN
}

\begin{abstract}
Kaugummi-Schnelltest für Entzündungen an Implantaten-- Bereits mehr als eine Million künstliche Zahnwurzeln werden Schätzungen zufolge in Deutschland pro Jahr implantiert. Doch bei bis zu 15 Prozent der Patienten kommt es nach einigen Jahren zu einer Mukositis oder sogar zu einer Periimplantitis.

Schlimmstenfalls werden dabei das weiche Gewebe und der Knochen rund um das Implantat zerstört.

Mithilfe eines aMMP-8-BiomarkerSchnelltests kann frühzeitig eine solche Entzündung feststellt werden: Das

Enzym aMMP-8 wird früh während der parodontalen Entzündung vom körpereigenen Immunsystem aktiviert. Durch den Nachweis hoher aMMP-8-Werte
\end{abstract}

kann parodontaler Gewebeabbau erkannt werden, noch bevor klinische Zeichen sichtbar werden.

Diesen Nachweis erbringt nun auch ein neu entwickelter Kaugummi. Dieser

\section{"EIN NEUER KAU- GUMMI ERSETZT DEN BIOMARKER- SCHNELLTEST AUS DEM LABOR。}

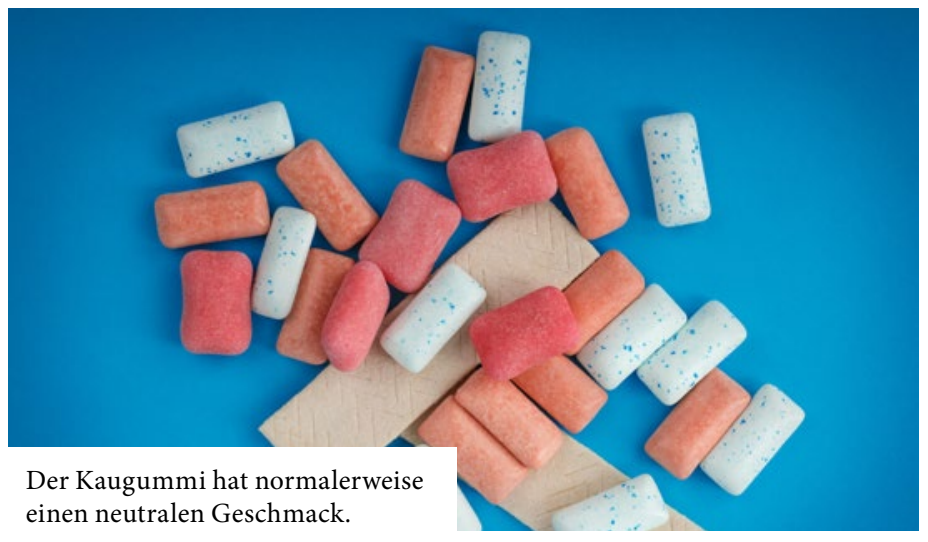

\section{WIEVIEL KRANKENPFLEGER VERDIENEN}

Entgeltatlas-- Krankenpfleger ohne Spezialisierung verdienen in Deutschland im Durchschnitt 3.240 Euro brutto im Monat, Fachkrankenpfleger mit Spezialisierung 3.629 Euro. Altenpfleger erhalten durchschnittlich 2.621 Euro im Monat und medizinische Fachangestellte 2.157 Euro. Diese Zahlen nennt aktuell der Entgeltatlas der Bundesagentur für Arbeit. Allerdings schwanken die Entgelte zwischen den Bundesländern deutlich. Bei den Krankenpflegern ohne Spezialisierung liegt das durchschnittliche Einkommen in Niedersachsen und in den östlichen Bundesländern inklusive Ber- lin zwischen 2.753 und 3.240 Euro. In den übrigen Bundesländern sind es zwischen 3.241 und 3.705 Euro. Bei den medizinischen Fachangestellten beläuft sich der Durchschnittsverdienst in Thüringen, Sachsen und Sachsen-Anhalt auf unter 1.800 Euro. In Brandenburg, Berlin, Mecklenburg-Vorpommern und Niedersachsen liegt das Durchschnittseinkommen unter 2.158 Euro und in den übrigen Bundesländern zwischen 2.158 und 2.627 Euro. Auch für die Altenpflege schwanken die Zahlen regional von unter 2.217 Euro bis 3.148 Euro. MAR könnte das Labor ersetzen und Kosten einsparen helfen, indem Patienten überall und jederzeit selbst den Test durchführen können, wie eine entsprechende Studie im Magazin Nature Communications vorschlägt. Entwickelt von Wissenschaftlern der Universität Würzburg fungiert die Substanz Denatonium, die bitterste bekannte Substanz, im Kaugummi als Sensor. Der Bitterstoff ist darin fest an ein Peptid gebunden, der Kaugummi hätte also normalerweise einen neutralen Geschmack. Auch der Speichel eines gesunden Menschen kann den Bitterstoff nicht vom Träger lösen. Dies ändert sich, wenn der Speichel das Enzym MMP-8 enthält. MAR

\section{ACHTUNG, MÄNNER, VOR ZU VIEL ZUCKER!}

Risiko von Angstzuständen-- Zu viel Zucker ist gerade für Männer gesundheitsschädigend, wie Forscher des University College London herausgefunden haben. Laut Dental Tribune wiesen die Wissenschaftler nach, dass Männer Angstzustände und Depressionen infolge von zu viel Zuckerkonsum entwickeln können. Für die Studie wurden mehr als 5.000 Männer und knapp 2.000 Frauen auf den Zusammenhang zwischen Zuckeraufnahme und möglichen Stimmungsstörungen untersucht. Das Ergebnis: Männer, die mehr als 67 Gramm Zucker pro Tag konsumierten, wiesen nach fünf Jahren ein um 23 Prozent erhöhtes Risiko für Stimmungsstörungen, wie etwa Depressionen, auf. Männer, die täglich weniger als $40 \mathrm{Gramm}$ zu sich nahmen, waren nicht gefährdet. Bei den Frauen war kein Zusammenhang zwischen erhöhtem Zuckerkonsum und psychischen Störungen festzustellen. MAR 